\title{
Time-of-Flight Mass Measurements of Neutron Rich Nuclides
}

\author{
Alfredo Estradé ${ }^{* a b c}$ Milan Matoš, ${ }^{a b}$ Matthew Amthor, ${ }^{a b c}$ Daniel Bazin, ${ }^{a}$ Ana \\ Becerril, ${ }^{a b c}$ Thom Elliot, ${ }^{a b c}$ Alexandra Gade, ${ }^{a c}$ Daniel Galaviz, ${ }^{a b}$ Giuseppe \\ Lorusso, ${ }^{a b c}$ Jorge Pereira, ${ }^{a b}$ Mauricio Portillo, ${ }^{a}$ Andrew Rogers, ${ }^{a b c}$ Hendrik \\ Schatz, ${ }^{a b c}$ Dan Shapira, ${ }^{d}$ Edward Smith, ${ }^{b e}$ Andreas Stolz, ${ }^{a}$ and Mark Wallace ${ }^{f}$ \\ ${ }^{a}$ National Superconducting Cyclotron Laboratory, Michigan State University \\ East Lansing, MI, USA \\ ${ }^{b}$ Joint Institute for Nuclear Astrophysics \\ ${ }^{c}$ Department of Physics and Astronomy, Michigan State University \\ East Lansing, MI, USA \\ ${ }^{d}$ Oak Ridge National Laboratory \\ Oak Ridge, TN, USA \\ ${ }^{e}$ The Ohio State University \\ Columbus, OH, USA \\ ${ }^{f}$ Los Alamos National Laboratory \\ Los Alamos, NM, USA \\ E-mail: estrade@nscl.msu.edu, matos@lsu.edu
}

\begin{abstract}
Nuclear masses of neutron-rich isotopes in the $Z \sim 20-30$ region have been measured by the time-of-flight technique at the National Superconducting Cyclotron Laboratory (NSCL). The time-of-flight technique has shown the potential to access nuclear masses very far from stability when applied at radioactive beam facilities like the NSCL. Such measurements would provide valuable information for astrophysical model calculations of processes involving exotic nuclides, for example the r-process, and electron captures in the crust of accreting neutron stars.
\end{abstract}

10th Symposium on Nuclei in the Cosmos

July 27 - August 12008

Mackinac Island, Michigan, USA

\footnotetext{
* Speaker.
} 


\section{Introduction and Motivation}

The time-of-flight (TOF) technique has been successful at measuring neutron-rich isotopes up to mass $\sim 75$ [1][2]. Nuclear masses in this region are relevant for several astrophysical processes. In the electron capture process proposed to take place in the crust of accreting neutron stars the extreme density conditions drive the composition of nuclear matter towards the neutron drip line [3]. The energy released by these capture reactions could provide a significant heat source that affects several features of accreting neutron stars (e.g. cooling curves, and X-ray superburst ignition depths). Ground state energies determine the depth at which subsequent electron captures become energetically possible. Nuclear masses also provide a key ingredient for the calculation of nucleosynthesis processes, such as charged particle reaction processes in the early stages of a type II supernovae explosion (see for example [4]). This process is believed to produce the seed nucleus for the subsequent r-process, and could also contribute a significant part of the nucleosynthetic yield of light mass isotopes from a supernovae. For these reasons, we have implemented the TOF mass measurement technique at the National Superconducting Cyclotron Laboratory (NSCL) where the intense radioactive beams available provide access to many isotopes of astrophysical interest [5].

\section{TOF-B $\rho$ mass measurements near the $\mathrm{N}=\mathbf{5 0}$ shell gap}

Recent progress in nuclear mass measurements has followed two main directions: improving the resolution of the measurements and extending their reach towards more unstable isotopes. This has been possible because of the many existing techniques to measure masses (see [6], [7] for recent reviews). Within this context, the TOF technique is a very sensitive one and can produce some of the measurements farther away from stability, although it offers a limited accuracy $(\sim 200 \mathrm{keV})[8]$. It can be applied to measure isotopes with count rates as low as $0.01 s^{-1}$, and it has practically no limitation on their half lives (TOF is typically $<1 \mu s$ ).

The mass is derived from the equation of motion of the isotopes through a magnetic system (the beamline), by measuring their TOF relative to that of known masses (calibration isotopes):

$$
B \rho=\frac{\gamma p}{q}=\frac{\gamma m_{0}}{q}\left(\frac{L}{T O F}\right)
$$

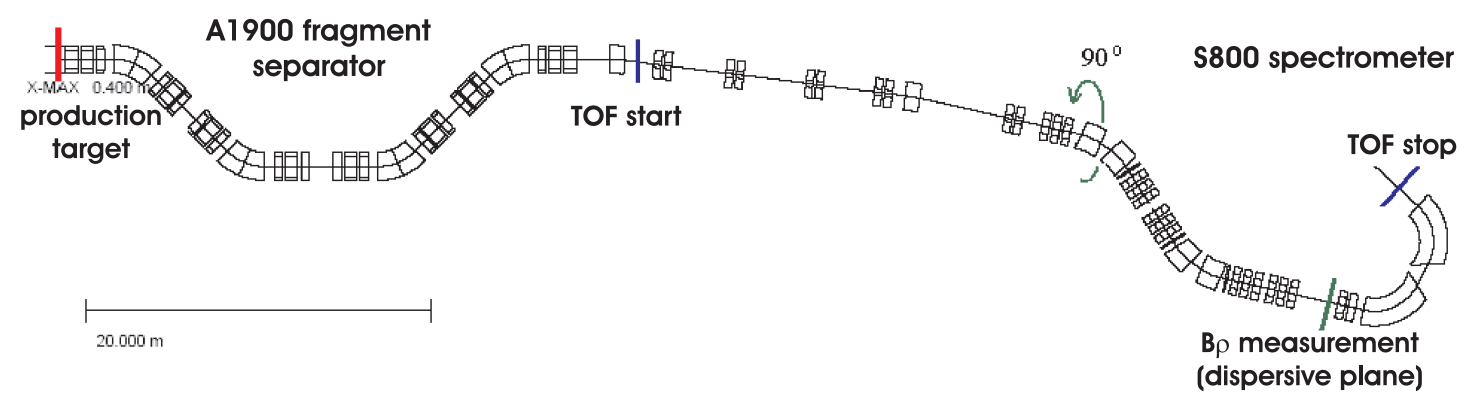

Figure 1: Experimental setup for TOF-B $\rho$ mass measurements at the NSCL. For a detailed description of the detector systems see [11]. 


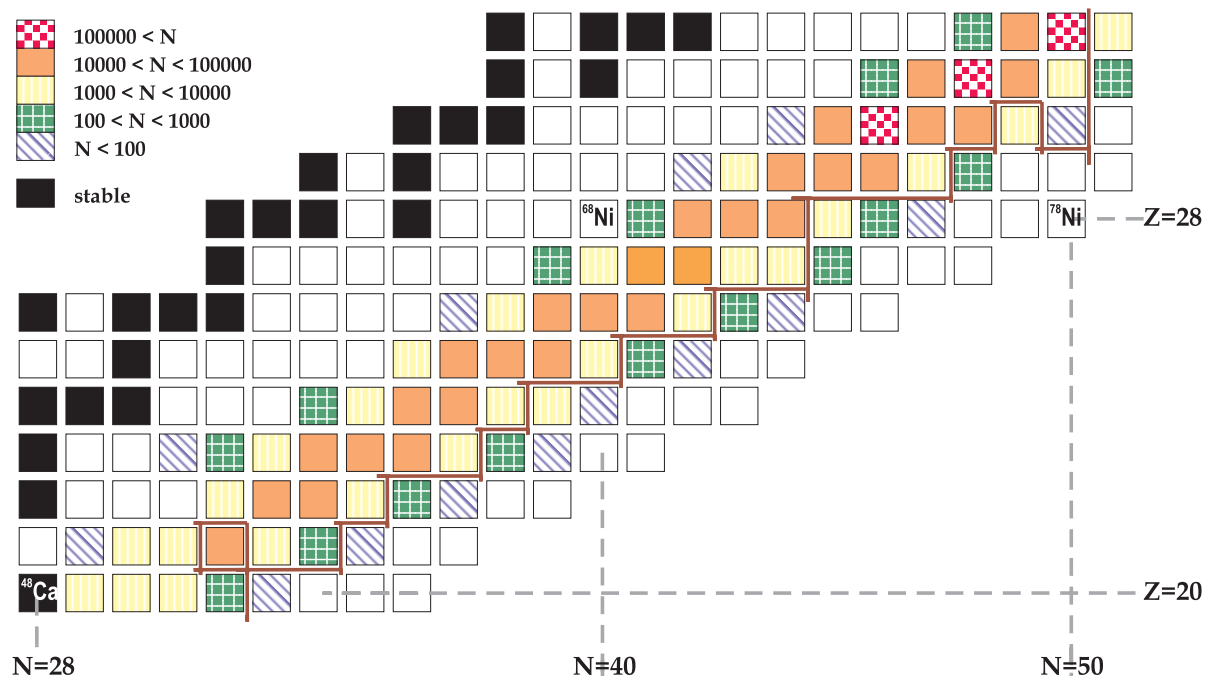

Figure 2: Region in the chart of the nuclides covered in our experiment. The solid line shows the limit of known masses according to the latest Atomic Mass Evaluation (AME2003 [12]). Isotopes are colored according to the number of observed events.

A central $B \rho$, or magnetic rigidity, is selected by the strength of the field of the beamline dipole magnets. Beamline systems usually have a finite $B \rho$ acceptance, and particles with different $B \rho$ will have different momenta and follow trajectories with slightly different path lengths. Therefore, in many cases, such as the present work, the $B \rho$ of each particle is measured (relative to that of a reference trajectory) to perform any necessary correction to the TOF.

At the NSCL we produced a beam of neutron-rich isotopes in the $\mathrm{Fe}$ region by fragmentation of a $100 \mathrm{MeV} / \mathrm{u}^{86} \mathrm{Kr}$ primary beam in a $\mathrm{Be}$ target. The TOF was measured with fast plastic scintillators over a 58 meter path from the exit of the fragment separator to the focal plane of the S800 spectrometer [9]. The $B \rho$ was measured with position sensitive microchannel plate (MCP) detectors [10] at the $\mathrm{S} 800$ dispersive plane, where $\mathrm{x}$-position is proportional to $B \rho$. We achieved a TOF resolution of $\sim 80 \mathrm{ps}$ for the determination of the TOF of each species [11]. Given typical TOFs of $\sim 500 n s$ it corresponds to a resolution of $(1-2) \times 10^{-4}$. Figure 1 shows the experimental setup, and figure 2 the region of our experiment.

\section{Results}

We corrected the measured TOF using a linear function in $B \rho$ to obtain the TOF for the central trajectories (figure 3). We used a calibration function that includes cubic terms in the particle's TOF and charge $(\mathrm{Z})$ in the expansion of 2.1, resulting in a total of 7 fit parameters. We fit this function to a set of 34 calibration isotopes, including masses from the latest Atomic Mass Evaluation (AME2003 [12]), as well as from recent penning trap measurements ([13], [14], [15]). As a measure of the goodness of fit figure 4 shows the difference between the calibration isotopes' masses evaluated with the calibration function and their previously measured values $\left(R M S_{\text {fit residuals }}=244 \mathrm{keV}\right)$. 

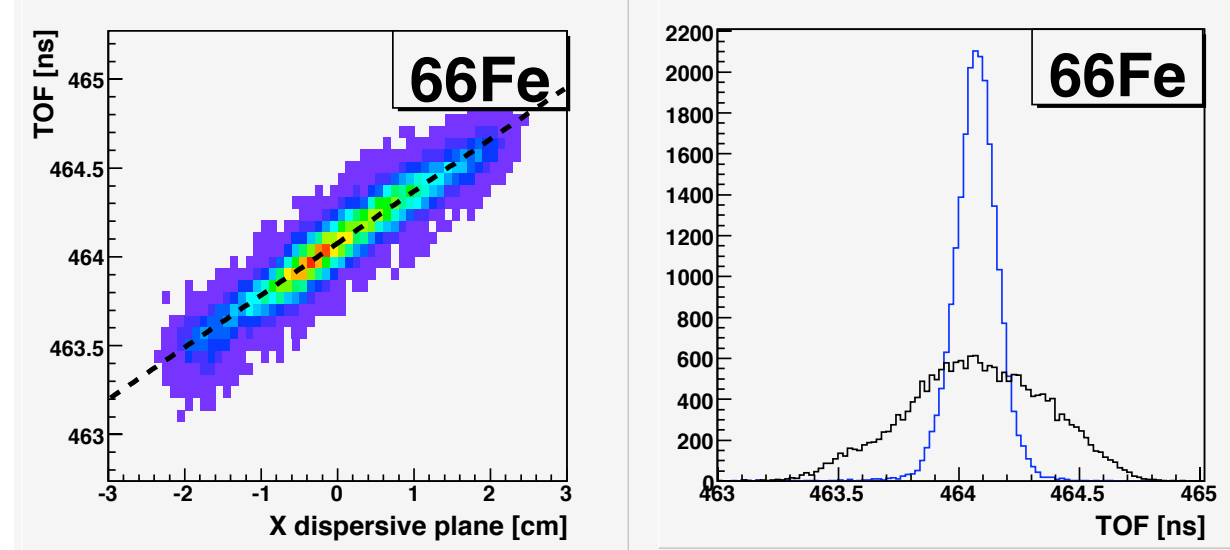

Figure 3: The left plot shows the relation between TOF and $x$-position $(B \rho)$ at the $S 800$ dispersive plane. The right figure shows how the resolution is improved for the $B \rho$-corrected TOF (blue/light line).

With this calibration function we studied the error that different variables in the experimental setup introduced in our measurement. Such systematic error was estimated as the $\sigma_{s y s}$ necessary to obtain a $\chi^{2}$ per degree of freedom of 1 for each data subset:

$$
\chi^{2}=\sum_{A, Z} \frac{\left(m_{A M E}-m_{\text {exp }}\right)^{2}}{\sigma_{\text {exp }}{ }^{2}+\sigma_{A M E}{ }^{2}+\sigma_{\text {sys }}^{2}}=n_{\text {calibration isotopes }}-n_{\text {fit parameters }}
$$

We explored the effect of: 1) temperature variations in the experimental vault, 2) correction to nonlinearities in the electronic modules (TAC-ADC), 3) voltage setting of MCP detectors, 4) measurement of TOF with respect to an independent clock, 5) method to correct TOF by $B \rho$. We learned that the response of the timing electronic modules is sensitive to the temperature and the method used to correct their nonlinearities, but using a clock that randomizes the measurement throughout the $\mathrm{ADC}$ range renders the TOF measurement more robust. The fit is also quite sensitive to the value of the slope used for the linear correction of the TOF by $B \rho$ (it should be noted that achieving a $200 \mathrm{keV}$ mass uncertainty requires determining the TOF with a sub-picosecond error).

\section{Conclusion}

We have performed a TOF-B $\rho$ mass measurement experiment of neutron-rich nuclei near the $\mathrm{N}=50$ shell gap at the NSCL. Preliminary analysis has shown we can achieve a mass resolution of $(1-2) \times 10^{-4}$, which corresponds to $\delta m \sim 150 \mathrm{keV}$ for $N \sim 5 \times 10^{3}$ events and $A \sim 60$. We used a calibration function that relies in the correction of the measured TOF by each particle's $B \rho$ to detect systematic effects in the experimental setup. It also provided a reasonable fit as evidenced by the fit residuals (figure 4). Work is in progress to obtain a more detailed error analysis, and we expect new and improved mass measurements for several nuclei in this region soon.

\section{References}

[1] H. Savajols, Hyperfine Interact. 132, 243 (2001). 


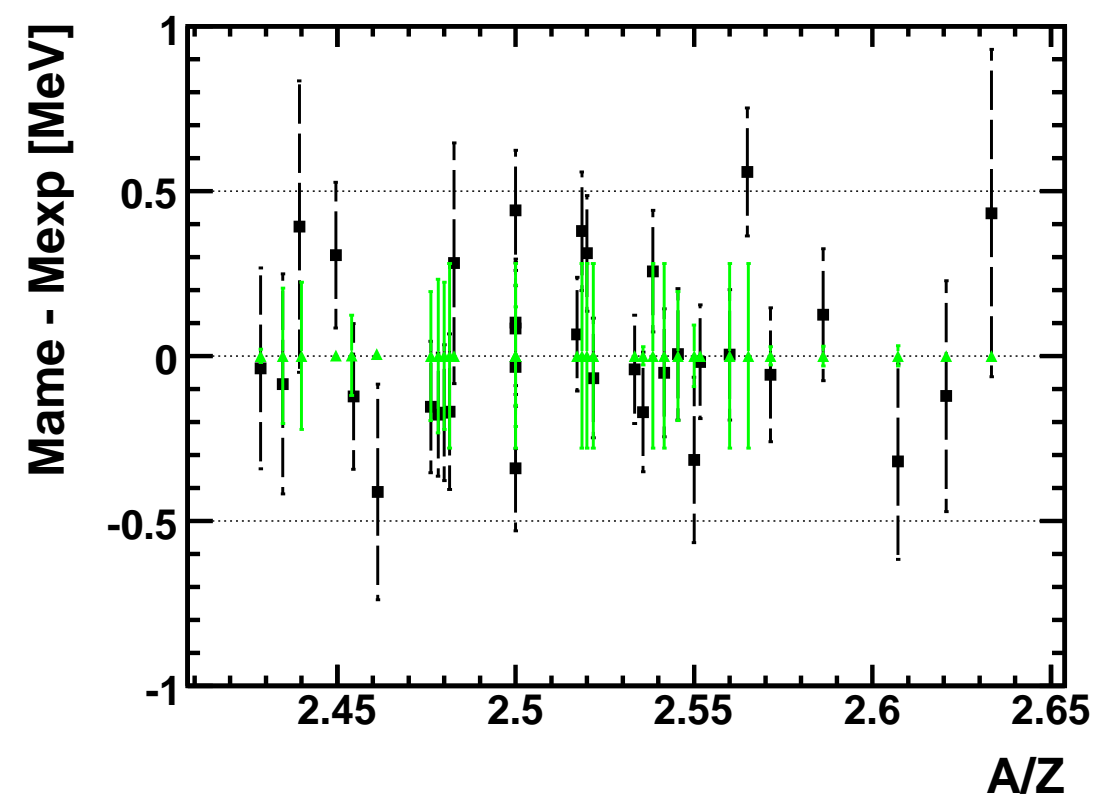

Figure 4: Deviation of the fit results (mass of calibration isotopes) from their published values as a function of the isotopes mass-to-charge ratio. The error bars for each calibrant represent our experimental uncertainty (dashed black line), and the uncertainty in the previous measurements is shown as error bars around the zero line (solid green line).

[2] J. M. Wouters, et al., Nucl. Instrum. Methods Phys. Res. B26, 286 (1987).

[3] S. Gupta, et al., Astrophys. J. 662, 1188 (2007).

[4] K. Farouqi, et al., in First Stars III, AIP Conf. Proc. 990, 309 (2008).

[5] A. Stolz, et al., Nucl. Instrum. Methods Phys. Res. B241, 858 (2005).

[6] K. Blaum, Phys. Rep. 425, 1 (2006).

[7] D. Lunney, J. M. Pearson, C. Thibault, Rev. Mod. Phys. 75, 1021 (2003).

[8] M. Matoš, in Capture Gamma Ray Spectroscopy and Related Topics, AIP Conf. Proc. 819, 164 (2005).

[9] D. Bazin, et al., Nucl. Instrum. Methods Phys. Res. B204, 629 (2000).

[10] D. Shapira, T. A. Lewis, and L.D. Hulett, Nucl. Instrum. Methods Phys. Res. A454, 409 (2000).

[11] A. Estradé, et al., in Proc. of Int. Symp. Nuclei in the Cosmos IX, PoS(NIC-IX) 092 (2006).

[12] G. Audi, A. H. Wapstra, and C. Thibault, Nucl. Phys. A729, 337 (2003).

[13] C. Guénaut, et al., Phys. Rev. C75, 044303 (2007).

[14] M. Block, et al., Phys. Rev. Lett. 100, 132501 (2008).

[15] S. Rahaman, et al., Eur. Phys. J. A34, 5 (2007). 